\title{
REGULARIZATION OF AN UNILATERAL OBSTACLE PROBLEM
}

\author{
Ahmed Addou ${ }^{1}$, E. BekKaye Mermri ${ }^{1}$ And Jamal Zahi ${ }^{1}$
}

\begin{abstract}
The aim of this article is to give a regularization method for an unilateral obstacle problem with obstacle $\psi$ and second member $f$, which generalizes the one established by the authors of [4] in case of null obstacle and a second member is equal to constant 1.
\end{abstract}

Mathematics Subject Classification. 35J85.

Received: May 10, 2001.

\section{INTRODUCTION}

Let $\Omega$ be a bounded domain of $\mathbb{R}^{n}$ with smooth boundary $\partial \Omega, g \in H^{1 / 2}(\partial \Omega)$ and $\psi \in H^{1}(\Omega)$. We consider the variational inequality problem - called unilateral obstacle problem -:

Find

$$
u \in K=\left\{v \in H^{1}(\Omega) ; v \geq \psi \text { a.e. in } \Omega, v=g \text { on } \partial \Omega\right\}
$$

such that

$$
\int_{\Omega} \nabla u \nabla(v-u) \mathrm{d} x+\langle f, v-u\rangle \geq 0 \quad \forall v \in K
$$

where $f \in H^{-1}(\Omega)$. It is well known that Problem (1-2) admits a unique solution (see [5]).

The aim of this article is to develop a regularization method for solving a non differentiable minimization problem which is equivalent to Problem (1-2). The idea of the regularization method is to approximate the non differentiable term by a sequence of differentiable ones depending on $\varepsilon>0, \varepsilon \rightarrow 0$. To establish this regularization we give a new formulation of the obstacle problem, which is the subject of Theorem 1 . We give three forms of regularization for which we establish the convergence result and a priori error estimates. Next by the duality method by conjugate functions (see [2]) we provide a posteriori error estimates which is desired for practical implementation for the regularization method.

This study is a generalization of an other one established by the authors of [4], where the obstacle $\psi$ is equal to zero and the second member $f$ is taken equal to the constant 1 .

Keywords and phrases. Regularization, obstacle, unilateral.

1 Department of Mathematics, Faculty of Sciences, University Mohammed I, Oujda, Morocco.

e-mail: zahi-j@sciences.univ-oujda.ac.ma 


\section{Formulation and Regularization of the Problem}

Let $\Omega$ be a bounded domain of $\mathbb{R}^{n}$, with smooth boundary $\partial \Omega$ and $g \in H^{1 / 2}(\partial \Omega)$, we denote by

$$
H_{g}^{1}(\Omega)=\left\{v \in H^{1}(\Omega) ; v=g \text { on } \partial \Omega\right\} .
$$

For $\psi$ an element of $H^{1}(\Omega)$ with $\psi \leq g$ on $\partial \Omega$, we set

$$
K_{\psi}=\left\{v \in H_{g}^{1}(\Omega): v \geq \psi \text { a.e. on } \Omega\right\}
$$

Let $f \in H^{-1}(\Omega)$, we assume that $f$ and $\psi$ verify the following hypothesis:

$$
f-\Delta \psi=F \in L^{2}(\Omega)
$$

We denote by $\langle.,$.$\rangle the duality pairing between H^{-1}(\Omega)$ and $H_{0}^{1}(\Omega)$, and $(.,$.$) the inner product of L^{2}(\Omega)$. Consider the following variational inequality problem:

$$
\left(P_{\psi}\right)\left\{\begin{array}{l}
\text { Find } u \in K_{\psi} \\
a(u, v-u)+\langle f, v-u\rangle \geq 0 \quad \text { for all } v \in K_{\psi},
\end{array}\right.
$$

where $a(.,$.$) is defined by$

$$
a(u, v)=\int_{\Omega} \nabla u . \nabla v, \quad u, v \in H^{1}(\Omega) .
$$

It is well-known that Problem $\left(P_{\psi}\right)$ admits a unique solution.

For all element $z \in L^{2}(\Omega)$ we denote

$$
z^{+}=\max \{z, o\} \text { and } z^{-}=\min \{z, o\}
$$

If $v \in H^{1}(\Omega)$, then we have $v^{+}, v^{-} \in H^{1}(\Omega)$ and

$$
a\left(v^{+}, v^{-}\right)=0
$$

In the sequel we use the same notation $g$ to designate an element of $H^{1 / 2}(\partial \Omega)$ and an element of $H^{1}(\Omega)$ which its trace on $\partial \Omega$ is $g$. We write the obstacle problem $\left(P_{\psi}\right)$ on a new form.

Theorem 1. $u$ is solution of Problem $\left(P_{\psi}\right)$ if and only if $w=u-g$ is solution of the following problem:

$$
(P)\left\{\begin{array}{l}
\text { Find } w \in H_{0}^{1}(\Omega), \\
a(w+g-\psi, v-w)+\varphi(v)-\varphi(w)+\left(F^{-}, v-w\right) \geq 0 \text { for all } v \in H_{0}^{1}(\Omega),
\end{array}\right.
$$

where $\varphi$ is the functional defined by

$$
\varphi(v)=\left(F^{+}, \phi(v+g-\psi)\right), \quad v \in H_{0}^{1}(\Omega)
$$

with

$$
\phi(t)=t^{+}, \quad t \in \mathbb{R} .
$$

Proof. From the general theory of variational inequalities (see [3]), Problem $(P)$ admits a unique solution, so it is sufficient to show that $w+g$ is a element of $K_{\psi}$, where $w$ is the solution of Problem $(P)$. Indeed, for $v=(w+g-\psi)^{+}-g+\psi \in H_{0}^{1}(\Omega)$, the inequality of $(P)$ becomes

$$
-a\left((w+g-\psi)^{-},(w+g-\psi)^{-}\right)+\varphi(w)-\varphi(w)-\left(F^{-},(v+g-\psi)^{-}\right) \geq 0 .
$$


Hence, from (3) we obtain

$$
a\left((w+g-\psi)^{-},(w+g-\psi)^{-}\right)=0,
$$

we deduce that $w+g-\psi \geq 0$, consequently $w+g \in K_{\psi}$.

It is easy to see that $u=w+g$ verify the inequality of Problem $(P)$, hence the proof is complete.

The functional $\varphi$ being non differentiable on $H_{0}^{1}(\Omega)$, we approximate it by a sequence of differentiable functionals, $\varphi_{\varepsilon}(v)=\int_{\Omega} F^{+} \phi_{\varepsilon}(v+g-\psi) \mathrm{d} x,(\varepsilon>0$, tends to 0$)$. The regularized problem is

$$
\left(P_{\varepsilon}\right)\left\{\begin{array}{l}
\text { Find } w_{\varepsilon} \in H_{0}^{1}(\Omega) \\
a\left(w_{\varepsilon}, v-w_{\varepsilon}\right)+\varphi_{\varepsilon}(v)-\varphi_{\varepsilon}\left(w_{\varepsilon}\right)+\left\langle l, v-w_{\varepsilon}\right\rangle \geq 0 \text { for all } v \in H_{0}^{1}(\Omega)
\end{array}\right.
$$

where

$$
\langle l, v\rangle=a(g-\psi, v)+\left(F^{-}, v\right) .
$$

Problems $(P)$ and $\left(P_{\varepsilon}\right)$ are, respectively, equivalent to

$$
u \in H_{g}^{1}(\Omega): a(u, v-u)+\tilde{\varphi}(v)-\tilde{\varphi}(u)+\int_{\Omega}\left(F^{-}+\Delta \psi\right)(v-u) \mathrm{d} x \geq 0 \quad \forall v \in H_{g}^{1}(\Omega),
$$

with

and

$$
\tilde{\varphi}(v)=\int_{\Omega} \phi(v-\psi) \mathrm{d} x, \quad v \in H_{g}^{1}(\Omega)
$$

$$
u_{\varepsilon} \in H_{g}^{1}(\Omega): a\left(u_{\varepsilon}, v-u_{\varepsilon}\right)+\tilde{\varphi}_{\varepsilon}(v)-\tilde{\varphi}_{\varepsilon}\left(u_{\varepsilon}\right)+\int_{\Omega}\left(F^{-}+\Delta \psi\right)\left(v-u_{\varepsilon}\right) \mathrm{d} x \geq 0 \quad \forall v \in H_{g}^{1}(\Omega)
$$

with

$$
\tilde{\varphi}_{\varepsilon}(v)=\int_{\Omega} F^{+} \phi_{\varepsilon}(v-\psi) \mathrm{d} x, \quad v \in H_{g}^{1}(\Omega) .
$$

There are many methods to construct sequences of differentiable approximations. In this article we take the sequence $\phi_{\varepsilon}$ verifying one of the following choices:

$$
\begin{array}{ll}
\mathrm{c} 1: & \phi_{\varepsilon}^{1}(t)= \begin{cases}t-\frac{\varepsilon}{2} & \text { if } t \geq \varepsilon \\
\frac{t^{2}}{2 \varepsilon} & \text { if } 0 \leq t \leq \varepsilon \\
0 & \text { if } t \leq 0 .\end{cases} \\
\mathrm{c} 2: & \phi_{\varepsilon}^{2}(t)= \begin{cases}t & \text { if } t \geq \varepsilon \\
\frac{1}{2}\left(\frac{t^{2}}{\varepsilon}+\varepsilon\right) & \text { if } 0 \leq t \leq \varepsilon \\
\frac{\varepsilon}{2} & \text { if } t \leq 0 .\end{cases} \\
\mathrm{c} 3: & \phi_{\varepsilon}^{3}(t)= \begin{cases}\sqrt{t^{2}+\varepsilon^{2}} & \text { if } t \geq 0 \\
\varepsilon & \text { if } t \leq 0 .\end{cases}
\end{array}
$$

With these choices Problem $\left(P_{\varepsilon}\right)$ admits a unique solution. To establish the convergence of Sequence $u_{\varepsilon}$ we need the following results (see [3]).

Lemma 1. Let $V$ be a Hilbert space, $a: V \times V \rightarrow \mathbb{R}$ a continuous, V-elliptic bilinear form, $j: V \rightarrow \mathbb{R}$ proper, non negative, convex, weakly continuous function and $f$ is a linear continuous form on $V$. Assume that $j_{\varepsilon}: V \rightarrow \mathbb{R},(\varepsilon>0)$, is a family of non negative convex weakly lower semi-continuous (l.s.c.) functions verifying

$$
j_{\varepsilon}(v) \rightarrow j(v) \quad \forall v \in V,
$$




$$
\text { If } u_{\varepsilon} \rightarrow u \text { weakly in } V \text { then we have } j(u) \leq \lim _{\varepsilon \rightarrow 0} \inf j_{\varepsilon}\left(u_{\varepsilon}\right) .
$$

Let $u, u_{\varepsilon} \in V$ be the solutions of the following variational inequalities:

$$
\begin{aligned}
a(u, v-u)+j(v)-j(u)+\langle f, v-u\rangle & \geq 0, \quad \forall v \in V, \\
a\left(u_{\varepsilon}, v-u_{\varepsilon}\right)+j_{\varepsilon}(v)-j_{\varepsilon}\left(u_{\varepsilon}\right)+\left\langle f, v-u_{\varepsilon}\right\rangle & \geq 0, \quad \forall v \in V,
\end{aligned}
$$

respectively. Then we have $u_{\varepsilon} \rightarrow u$ in $V$ when $\varepsilon \rightarrow 0$.

Lemma 2. Assume that

$$
j(v)=\int_{\Omega} \phi(v) \mathrm{d} x, j_{\varepsilon}(v)=\int_{\Omega} \phi_{\varepsilon}(v) \mathrm{d} x
$$

and $j$ is weakly l.s.c. If

$$
\phi_{\varepsilon}(t) \rightarrow \phi(t) \text { uniformly in } t \text {, as } \varepsilon \rightarrow 0 \text {, }
$$

then (6) and (7) are verified.

We notice that if

$$
\left|\phi_{\varepsilon}(t)-\phi(t)\right| \leq c \varepsilon \quad \forall t \in \mathbb{R},
$$

then (8) is verified. Since the functions $\phi_{\varepsilon}^{j}, j=1,2,3$ verify the inequality (9), then we have the convergence $w_{\varepsilon} \rightarrow w$ in $H_{0}^{1}(\Omega)$.

Taking $v=w_{\varepsilon}($ resp. $v=w)$ in the inequality of Problem $(P)$ (resp. $\left(P_{\varepsilon}\right)$ ), we obtain

$$
a\left(w-w_{\varepsilon}, w-w_{\varepsilon}\right) \leq \varphi\left(w_{\varepsilon}\right)-\varphi_{\varepsilon}\left(w_{\varepsilon}\right)+\varphi_{\varepsilon}(w)-\varphi(w) .
$$

Consequently, we obtain the following a priori estimate

$$
\left\|w-w_{\varepsilon}\right\|_{H_{0}^{1}(\Omega)} \leq\left(2 c \int_{\Omega} F^{+}\right)^{\frac{1}{2}} \sqrt{\varepsilon} .
$$

\section{A-POSTERIORI ERROR ESTIMATES}

In this section we use the duality method by conjugate functions in order to derive the a posteriori error estimates of solutions of approximate problems. We need the following preliminary results (see [2])

Let $V$ and $V^{*}$ (resp. $Y$ and $Y^{*}$ ) two topological vector spaces and $\langle., .\rangle_{V}$ (resp. $\langle.,\rangle_{Y}$ ) denotes the duality pairing between $V^{*}$ and $V$ (resp. $Y^{*}$ and $Y$ ). Let $\varphi$ be a function from $V$ to $\overline{\mathbb{R}}=\mathbb{R} \cup\{-\infty,+\infty\}$, its conjugate function is defined by

$$
\varphi\left(v^{*}\right)=\sup _{v \in V}\left\langle v^{*}, v\right\rangle_{V}-\varphi(v), \quad v^{*} \in V^{*} .
$$

Assume there exists a continuous linear operator $L$ from $V$ to $Y, L \in \mathcal{L}(V, Y)$, with transpose $L^{*} \in \mathcal{L}\left(Y^{*}, V^{*}\right)$. Let $J$ be a function from $V \times Y$ to $\overline{\mathbb{R}}$. We consider the following minimization problem:

$$
u \in V, \quad J(u, L u)=\inf _{v \in V} J(v, L v) .
$$

The conjugate function of $J$ is given by

$$
J^{*}\left(y^{*}, v^{*}\right)=\sup _{v \in V, y \in Y}\left\{\left\langle v^{*}, v\right\rangle_{V}+\left\langle y^{*}, y\right\rangle_{Y}-J(v, y)\right\}
$$


Theorem 2. Assume that $V$ is a reflexive Banach space and $Y$ a normed vector space. Let $J: V \times Y \rightarrow \overline{\mathbb{R}}$ be a proper l.s.c. strictly convex function verifying:

(i) $\exists u_{0} \in V$, such that $J\left(u_{0}, L u_{0}\right)<\infty$ and $y \rightarrow J\left(u_{0}, y\right)$ is continuous at $L u_{0}$.

(ii) $J(v, L v) \rightarrow+\infty$, as $\|v\|_{V} \rightarrow+\infty, v \in V$.

Then Problem (10) admits a unique solution and

$$
J(u, L u)=\inf _{v \in V} J(v, L v)=-\sup _{y^{*} \in Y^{*}} J^{*}\left(-y^{*}, L^{*} y^{*}\right)
$$

Let $\Omega$ be an open subset of $\mathbb{R}^{N}, g: \Omega \times \mathbb{R}^{n} \rightarrow \mathbb{R}$ be the Carathéodory function i.e., $\forall s \in \mathbb{R}^{n}, x \rightarrow g(x, s)$ is a measurable function and for almost all $x \in \Omega$, the function $s \rightarrow g(x, s)$ is continuous. Then the conjugate function of

$$
G(v)=\int_{\Omega} g(x, v(x)) \mathrm{d} x
$$

(assuming $G$ is well defined over some a function space $V$ ) is

$$
G^{*}\left(v^{*}\right)=\int_{\Omega} g^{*}\left(x, v^{*}(x)\right) \mathrm{d} x, \quad \forall v^{*} \in V^{*}
$$

where

$$
g^{*}(x, y)=\sup _{s \in \mathbb{R}^{N}}\{y s-g(x, s)\}
$$

For Problem $(P)$ we take

$$
\begin{aligned}
V & =H^{1}(\Omega), \quad Y=Y^{*}=\left(L^{2}(\Omega)\right)^{n} \times L^{2}(\Omega) \\
L v & =(\nabla v, v) \\
J(v, L v) & =H(v)+G(L v) \\
H(v) & =\left\{\begin{array}{cc}
0 & \text { if } v=g \text { on } \partial \Omega \\
+\infty & \text { otherwise }
\end{array}\right. \\
G(y) & =\int_{\Omega} \frac{1}{2}\left|y_{1}\right|^{2}+F^{+}\left(y_{2}-\psi\right)^{+}+F^{-} y_{2}+\Delta \psi y_{2}
\end{aligned}
$$

( Furthermore we assume that $\psi \geq 0$ )

where $y=\left(y_{1}, y_{2}\right)$ with $y_{1} \in\left(L^{2}(\Omega)\right)^{n}$ and $y_{2} \in L^{2}(\Omega)$. A similar notation is used for $y^{*} \in Y^{*}$. So the obstacle problem $(P)$ can be rewritten in the form (10). To apply Theorem 2, we compute the conjugate of the functional $J$.

Lemma 3. Let $h$ be a function defined by

$$
\begin{aligned}
h: \mathbb{R} & \rightarrow \mathbb{R} \\
s & \mapsto a s+b(s-t)^{+}
\end{aligned}
$$

where $a, b$ and $t$ are constants with $b \geq 0$, then the conjugate function $h^{*}$ of $h$ is given by

$$
h^{*}\left(s^{*}\right)= \begin{cases}t\left(s^{*}-a\right) & \text { if } a \leq s^{*} \leq a+b \\ +\infty & \text { otherwise. }\end{cases}
$$

If $t \geq 0$ then we have

$$
0 \leq h^{*}\left(s^{*}\right) \leq t b .
$$


Proof. We have

It is easy to check that

$$
\begin{aligned}
h^{*}\left(s^{*}\right) & =\sup _{s \in \mathbb{R}}\left\{s s^{*}-h(s)\right\} \\
& =\sup _{s \in \mathbb{R}}\left\{s s^{*}-a s-b(s-t)^{+}\right\} \\
& =\max \left\{\sup _{s \geq t}\left\{s s^{*}-a s-b s+b t\right\}, \sup _{s \leq t}\left\{s s^{*}-a s\right\}\right\} \\
& =\max \left\{\sup _{s \geq t}\left\{s\left(s^{*}-a-b\right)+b t\right\}, \sup _{s \leq t}\left\{s\left(s^{*}-a\right)\right\}\right\}
\end{aligned}
$$

Hence the proof is complete.

$$
h^{*}\left(s^{*}\right)= \begin{cases}t\left(s^{*}-a\right) & \text { si } a \leq s^{*} \leq a+b \\ +\infty & \text { otherwise }\end{cases}
$$

We have

$$
J^{*}\left(-y^{*}, L^{*} y^{*}\right)=H^{*}\left(L^{*} y^{*}\right)+G^{*}\left(-y^{*}\right)
$$

where

$$
\begin{aligned}
H^{*}\left(L^{*} y^{*}\right) & =\sup _{v \in H^{1}(\Omega)}\{\langle L v, y\rangle-H(v)\} \\
& =\sup _{v \in H_{g}^{1}(\Omega)} \int_{\Omega}\left(\nabla v y_{1}^{*}+v y_{2}^{*}\right) \mathrm{d} x \\
& =\int_{\Omega}\left(\nabla g y_{1}^{*}+g y_{2}^{*}\right) \mathrm{d} x+\sup _{v \in H_{0}^{1}(\Omega)} \int_{\Omega}\left(\nabla v y_{1}^{*}+v y_{2}^{*}\right) \mathrm{d} x \\
& = \begin{cases}\int_{\Omega}\left(\nabla g y_{1}^{*}+g y_{2}^{*}\right) \mathrm{d} x & \text { if }-\operatorname{div} y_{1}^{*}+y_{2}^{*}=0 \text { dans } \Omega \\
\infty & \text { otherwise }\end{cases}
\end{aligned}
$$

and

$$
\begin{aligned}
G^{*}\left(-y^{*}\right) & =\sup _{y \in Y}\left\{\left\langle-y^{*}, y\right\rangle-G(y)\right\} \\
& =\sup _{y \in Y} \int_{\Omega}\left(-y_{1}^{*} y_{1}-y_{2}^{*} y_{2}-\frac{1}{2}\left|y_{1}\right|^{2}-F^{+}\left(y_{2}-\psi\right)^{+}-F^{-} y_{2}-\Delta \psi y_{2}\right) \mathrm{d} x
\end{aligned}
$$

and from Lemma 3 we obtain

$$
G^{*}\left(-y^{*}\right)= \begin{cases}\leq \int_{\Omega}\left(\frac{1}{2}\left|y_{1}^{*}\right|^{2}+F^{+} \psi\right) \mathrm{d} x & \text { if }-f \leq y_{2}^{*} \leq F^{+}-f \\ \infty & \text { otherwise. }\end{cases}
$$

Hence

$$
J^{*}\left(-y^{*}, L^{*} y^{*}\right)= \begin{cases}\leq \int_{\Omega}\left(\nabla g y_{1}^{*}+g y_{2}^{*}+\frac{1}{2}\left|y_{1}^{*}\right|^{2}+F^{+} \psi\right) \mathrm{d} x & \text { if } \operatorname{div} y_{1}^{*}+y_{2}^{*}=0 \\ \infty & \text { and }-f \leq y_{2}^{*} \leq F^{+}-f \\ & \text { otherwise }\end{cases}
$$

We have

$$
\begin{aligned}
J\left(u_{\varepsilon}, L u_{\varepsilon}\right)-J(u, L u)= & \int_{\Omega} \frac{1}{2}\left|\nabla u_{\varepsilon}\right|^{2}-\frac{1}{2}|\nabla u|^{2}+F^{+}\left(u_{\varepsilon}-\psi\right)^{+}-F^{+}(u-\psi)^{+} \\
& +F^{-} u_{\varepsilon}-F^{-} u+\Delta \psi u_{\varepsilon}-\Delta \psi u .
\end{aligned}
$$

Using (4), with $v=u_{\varepsilon}$, we obtain

$$
J\left(u_{\varepsilon}, L u_{\varepsilon}\right)-J(u, L u) \geq \frac{1}{2}\left\|\nabla\left(u_{\varepsilon}-u\right)\right\|_{L^{2}(\Omega)}^{2} .
$$


Applying Theorem 2 and using (11), we have

$$
\begin{aligned}
J\left(u_{\varepsilon}, L u_{\varepsilon}\right)-J(u, L u) \leq & \int_{\Omega}\left(\frac{1}{2}\left|\nabla u_{\varepsilon}\right|^{2}+F^{+}\left(u_{\varepsilon}-\psi\right)^{+}+F^{-} u_{\varepsilon}+\Delta \psi u_{\varepsilon}\right. \\
& \left.+\nabla g y_{1}^{*}+g y_{2}^{*}+\frac{1}{2}\left|y_{1}^{*}\right|^{2}+F^{+} \psi\right) \mathrm{d} x
\end{aligned}
$$

$\forall y^{*}=\left(y_{1}^{*}, y_{2}^{*}\right) \in Q^{*}$, with $-\operatorname{div} y_{1}^{*}+y_{2}^{*}=0$ and $-f \leq y_{2}^{*} \leq F^{+}-f$ a.e. in $\Omega$.

Since $\phi_{\varepsilon}$ is differentiable the inequality (5) is equivalent to

$$
u_{\varepsilon} \in H_{g}^{1}(\Omega): a\left(u_{\varepsilon}, v\right)+\int_{\Omega}\left(F^{+} \phi_{\varepsilon}^{\prime}\left(u_{\varepsilon}-\psi\right)+F^{-}+\Delta \psi\right) v \mathrm{~d} x=0 . \quad \forall v \in H_{0}^{1}(\Omega) .
$$

Hence $u_{\varepsilon}$ verifies the following Dirichlet problem:

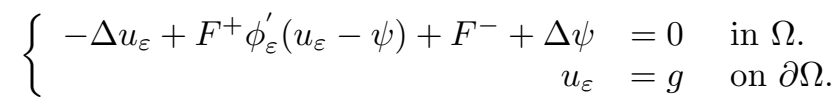

If we take

Then we have

$$
y_{1}^{*}=-\nabla u_{\varepsilon} \text { and } y_{2}^{*}=-\left(F^{+} \phi_{\varepsilon}^{\prime}\left(u_{\varepsilon}-\psi\right)+F^{-}+\Delta \psi\right) \text {. }
$$

$$
-\operatorname{div} y_{1}^{*}+y_{2}^{*}=0 \text { and }-f \leq y_{2}^{*} \leq F^{+}-f .
$$

Therefore, we have the a posteriori estimate

$$
\begin{aligned}
\frac{1}{2}\left\|\nabla\left(u_{\varepsilon}-u\right)\right\|_{L^{2}(\Omega) \leq}^{2} \leq & \int_{\Omega}\left(\nabla u_{\varepsilon} \nabla\left(u_{\varepsilon}-g\right)+F^{+}\left(u_{\varepsilon}-\psi\right)^{+}+F^{-} u_{\varepsilon}+\Delta \psi u_{\varepsilon}\right. \\
& \left.-g\left(F^{+} \phi_{\varepsilon}^{\prime}\left(u_{\varepsilon}-\psi\right)+F^{-}+\Delta \psi\right)+F^{+} \psi\right) \mathrm{d} x .
\end{aligned}
$$

Taking $v=u_{\varepsilon}-g \in H_{0}^{1}(\Omega)$ in (12), we obtain

$$
\int_{\Omega} \nabla u_{\varepsilon} \nabla\left(u_{\varepsilon}-g\right) \mathrm{d} x+\int_{\Omega}\left(F^{+} \phi_{\varepsilon}^{\prime}\left(u_{\varepsilon}-\psi\right)+F^{-}+\Delta \psi\right)\left(u_{\varepsilon}-g\right) \mathrm{d} x=0 .
$$

The estimate (13) becomes

$$
\frac{1}{2}\left\|\nabla\left(u_{\varepsilon}-u\right)\right\|_{L^{2}(\Omega)}^{2} \leq \int_{\Omega}\left(F^{+}\left(u_{\varepsilon}-\psi\right)^{+}+F^{-} u_{\varepsilon}+\Delta \psi u_{\varepsilon}-\left(F^{+} \phi_{\varepsilon}^{\prime}\left(u_{\varepsilon}-\psi\right)+F^{-}+\Delta \psi\right) u_{\varepsilon}+F^{+} \psi\right) \mathrm{d} x .
$$

Hence we obtain the a posteriori error estimates.

For choices $\mathrm{c} 1$ and $\mathrm{c} 2$, we have

The a posteriori error estimate is

$$
\phi_{\varepsilon}^{\prime}(t)= \begin{cases}1 & \text { if } t \geq \varepsilon \\ \frac{t}{\varepsilon} & \text { if } 0 \leq t \leq \varepsilon \\ 0 & \text { if } t \leq 0\end{cases}
$$

$$
\frac{1}{2}\left\|\nabla\left(u_{\varepsilon}-u\right)\right\|_{L^{2}(\Omega)}^{2} \leq \int_{\left[0 \leq u_{\varepsilon}-\psi \leq \varepsilon\right]} F^{+} u_{\varepsilon}\left(1-\frac{u_{\varepsilon}-\psi}{\varepsilon}\right) \mathrm{d} x+\int_{\left[u_{\varepsilon}-\psi<0\right]} F^{+} \psi \mathrm{d} x .
$$

For choice c3, we have

$$
\phi_{\varepsilon}^{\prime}(t)= \begin{cases}\frac{t}{\sqrt{t^{2}+\varepsilon^{2}}} & \text { if } t \geq 0 \\ 0 & \text { if } t \leq 0\end{cases}
$$


The a posteriori error estimate is

$$
\frac{1}{2}\left\|\nabla\left(u_{\varepsilon}-u\right)\right\|_{L^{2}(\Omega)}^{2} \leq \int_{\left[u_{\varepsilon}-\psi \geq 0\right]} F^{+} u_{\varepsilon}\left(1-\frac{u_{\varepsilon}-\psi}{\sqrt{\left(u_{\varepsilon}-\psi\right)^{2}+\varepsilon^{2}}}\right) \mathrm{d} x+\int_{\left[u_{\varepsilon}-\psi<0\right]} F^{+} \psi \mathrm{d} x .
$$

In particular, when $\psi=0$ we find

$$
\begin{aligned}
& \frac{1}{2}\left\|\nabla\left(u_{\varepsilon}-u\right)\right\|_{L^{2}(\Omega)}^{2} \leq \int_{\left[0 \leq u_{\varepsilon} \leq \varepsilon\right]} f^{+} u_{\varepsilon}\left(1-\frac{u_{\varepsilon}}{\varepsilon}\right) \mathrm{d} x \\
& \frac{1}{2}\left\|\nabla\left(u_{\varepsilon}-u\right)\right\|_{L^{2}(\Omega)}^{2} \leq \int_{\left[u_{\varepsilon} \geq 0\right]} f^{+} u_{\varepsilon}\left(1-\frac{u_{\varepsilon}}{\sqrt{u_{\varepsilon}^{2}+\varepsilon^{2}}}\right) \mathrm{d} x
\end{aligned}
$$

respectively.

\section{A-POSTERIORI ERROR ESTIMATES FOR REGUlaRIZED DiSCRETE PROBLEM}

Let $V_{h}$ be a finite element space approximating $H^{1}(\Omega)$, let $V_{0 h}$ be the finite element subspace of $V_{h}$ consisting of all element of $V_{h}$ which are zero on the boundary of the domain. We have $V_{0 h} \subset H_{0}^{1}(\Omega)$. Assume the boundary function $g$ can be represented exactly by a function from $V_{h}$. Then, a finite element solution $u_{h} \in V_{h}$ for the obstacle problem $(P)$ is determined from the following problem:

$$
\left(P_{h}\right)\left\{\begin{array}{l}
u_{h} \in V_{h}, u_{h}=g \text { on } \partial \Omega \\
a\left(u_{h}, v_{h}-u_{h}\right)+\left(F^{+},\left(u_{h}-\psi\right)^{+}-\left(v_{h}-\psi\right)^{+}\right)+\left(F^{-}-\Delta \psi, v_{h}-u_{h}\right) \geq 0 \\
\forall v_{h} \in V_{h}, v_{h}=g \text { on } \partial \Omega
\end{array}\right.
$$

If we set $u_{0 h}=u_{h}-g$, then $u_{0 h}$ is the solution of the problem

$$
\left(P_{0 h}\right)\left\{\begin{array}{l}
u_{0 h} \in V_{0 h} \\
a\left(u_{0 h}, v_{h}-u_{0 h}\right)+\varphi\left(v_{h}\right)-\varphi\left(u_{0 h}\right)+\left\langle l, v_{h}-u_{0 h}\right\rangle \geq 0 \quad \forall v_{h} \in V_{0 h} .
\end{array}\right.
$$

We can proceed similarly as in [3] to prove the convergence of the finite element approximations and to have a priori error estimates.

The regularized problem of $\left(P_{0 h}\right)$ is

$$
\left(P_{0 h, \varepsilon}\right)\left\{\begin{array}{l}
u_{0 h, \varepsilon} \in V_{0 h, \varepsilon} \\
a\left(u_{0 h, \varepsilon}, v_{h}-u_{0 h, \varepsilon}\right)+\varphi_{\varepsilon}\left(v_{h}\right)-\varphi_{\varepsilon}\left(u_{0 h, \varepsilon}\right)+\left\langle l, v_{h}-u_{0 h, \varepsilon}\right\rangle \geq 0 \quad \forall v_{h} \in V_{0 h} .
\end{array}\right.
$$

We can similarly prove that $\left(P_{0 h, \varepsilon}\right)$ have unique solutions and their solution converge to corresponding solution of Problem $\left(P_{0 h}\right)$. By the duality theory on the discrete problems we prove the following a posteriori error estimates.

For choices $\mathrm{c} 1$ and $\mathrm{c} 2$, the a posteriori error estimate is

$$
\frac{1}{2}\left\|\nabla\left(u_{h, \varepsilon}-u_{h}\right)\right\|_{L^{2}(\Omega)}^{2} \leq \int_{\left[0 \leq u_{h, \varepsilon}-\psi \leq \varepsilon\right]} F^{+} u_{h, \varepsilon}\left(1-\frac{u_{h, \varepsilon}-\psi}{\varepsilon}\right) \mathrm{d} x+\int_{\left[u_{h, \varepsilon}-\psi<0\right]} F^{+} \psi \mathrm{d} x .
$$

For choice $\mathrm{c} 3$, the a posteriori error estimate is

$$
\frac{1}{2}\left\|\nabla\left(u_{h, \varepsilon}-u\right)\right\|_{L^{2}(\Omega)}^{2} \leq \int_{\left[u_{h, \varepsilon}-\psi \geq 0\right]} F^{+} u_{\varepsilon, h}\left(1-\frac{u_{h, \varepsilon}-\psi}{\sqrt{\left(u_{h, \varepsilon}-\psi\right)^{2}+\varepsilon^{2}}}\right) \mathrm{d} x+\int_{\left[u_{h, \varepsilon}-\psi<0\right]} F^{+} \psi \mathrm{d} x .
$$


In particular, when $\psi=0$ we find

$$
\begin{aligned}
\frac{1}{2}\left\|\nabla\left(u_{h, \varepsilon}-u\right)\right\|_{L^{2}(\Omega)}^{2} & \leq \int_{\left[0 \leq u_{h, \varepsilon} \leq \varepsilon\right]} f^{+} u_{h, \varepsilon}\left(1-\frac{u_{h, \varepsilon}}{\varepsilon}\right) \mathrm{d} x \\
\frac{1}{2}\left\|\nabla\left(u_{h, \varepsilon}-u\right)\right\|_{L^{2}(\Omega)}^{2} & \leq \int_{\left[u_{h, \varepsilon} \geq 0\right]} f^{+} u_{h, \varepsilon}\left(1-\frac{u_{h, \varepsilon}}{\sqrt{u_{h, \varepsilon}^{2}+\varepsilon^{2}}}\right) \mathrm{d} x
\end{aligned}
$$

respectively.

\section{REFERENCES}

[1] A. Addou and E.B. Mermri, Sur une méthode de résolution d'un problème d'obstacle. Math-Recherche 63 Applications 2 (2000) 59-69.

[2] I. Ekeland and R. Temam, Analyse convexe et problèmes variationnels. Gauthier-Villars, Eds., Paris, Brussels, Montreal (1974).

[3] R. Glowinski, J.-L. Lions and R. Trémolières, Numerical Analysis of Variational Inequalities. North-Holland Publishing Company, Amsterdam, New York, Oxford (1981).

[4] H. Huang, W. Han and J. Zhou, The regularisation method for an obstacle problem. Numer. Math. 69 (1994) $155-166$.

[5] D. Kinderlehrer and G. Stampacchia, An Introduction to Variational Inequalities and their Applications. Academic Press, New York (1980).

To access this journal online:

www.edpsciences.org 